\title{
gु
}

\section{Scattering of Massless Particles in Arbitrary Dimensions}

\author{
Freddy Cachazo, ${ }^{1, *}$ Song He, ${ }^{1,2, \dagger}$ and Ellis Ye Yuan ${ }^{1,3, *}$ \\ ${ }^{1}$ Perimeter Institute for Theoretical Physics, Waterloo, Ontario N2L 2Y5, Canada \\ ${ }^{2}$ School of Natural Sciences, Institute for Advanced Study, Princeton, New Jersey 08540, USA \\ ${ }^{3}$ Department of Physics \& Astronomy, University of Waterloo, Waterloo, Ontario N2L 3G1, Canada
}

(Received 28 July 2014; published 20 October 2014)

\begin{abstract}
We present a compact formula for the complete tree-level $S$-matrix of pure Yang-Mills and gravity theories in arbitrary spacetime dimensions. The new formula for the scattering of $n$ particles is given by an integral over the positions of $n$ points on a sphere restricted to satisfy a dimension-independent set of equations. The integrand is constructed using the reduced Pfaffian of a $2 n \times 2 n$ matrix, $\Psi$, that depends on momenta and polarization vectors. In its simplest form, the gravity integrand is a reduced determinant which is the square of the Pfaffian in the Yang-Mills integrand. Gauge invariance is completely manifest as it follows from a simple property of the Pfaffian.
\end{abstract}

Introduction.-In a recent work [1], we pointed out the existence of equations connecting the space of kinematic invariants of $n$ massless particles in any dimension and that of the positions of $n$ points on a sphere. The equations are given by

$$
\sum_{b \neq a} \frac{s_{a b}}{\sigma_{a}-\sigma_{b}}=0 \quad \text { for } a \in\{1,2, \ldots, n\}
$$

where $s_{a b}=\left(k_{a}+k_{b}\right)^{2}=2 k_{a} \cdot k_{b}$, and $\sigma_{c}$ is the position of the $c$ th puncture. Motivated by some remarkable properties of these equations, namely, their connection to general kinematic invariants and Kawai-Lewellen-Tye (KLT) orthogonality [2], it was proposed that they are the backbone of the tree-level $S$-matrix of massless particles in any dimension and were called the scattering equations.

The scattering equations are invariant under $\operatorname{SL}(2, \mathbb{C})$ transformations when external vectors satisfy momentum conservation. They already made an appearance in previous literature in [3-6], and, in particular, in Ref. [7] by one of the authors in studying fundamental Bern-Carrasco-Johansson (BCJ) relations [8] in four dimensions [9]. The validity of the BCJ relations for gauge-theoretical amplitudes in any dimension $[8,10,11]$ also provides an important piece of evidence for the universal relevance of the scattering equations.

In Ref. [1], we also proposed the existence of formulas for the complete $S$-matrix of Yang-Mills and gravity theories in any dimension. In this Letter, we provide the explicit construction of such formulas.

Preliminaries.-The first step towards the construction of formulas in any spacetime dimension is that of the measure. Given that only $n-3$ of the $n$ scattering equations are linearly independent, one has to find a way of imposing their support in a permutation-invariant manner. This is achieved by noticing that

$$
\prod_{a}^{\prime} \delta\left(\sum_{b \neq a} \frac{s_{a b}}{\sigma_{a b}}\right):=\sigma_{i j} \sigma_{j k} \sigma_{k i} \prod_{a \neq i, j, k} \delta\left(\sum_{b \neq a} \frac{s_{a b}}{\sigma_{a b}}\right)
$$

is independent of the choice $\{i, j, k\}$ and, hence, permutation invariant. Here and in the rest of this Letter, $\sigma_{a b}:=\sigma_{a}-\sigma_{b}$.

Let us denote the $n$-gluon partial amplitude with the canonical ordering $1,2, \ldots, n$ as $A_{n}$ and the $n$-graviton amplitude as $M_{n}$. It is now natural to propose the following formulations of their $S$ matrices:

$$
\begin{aligned}
& A_{n}=\int \frac{d^{n} \sigma}{\operatorname{volSL}(2, \mathbb{C})} \prod_{a}{ }^{\prime} \delta\left(\sum_{b \neq a} \frac{s_{a b}}{\sigma_{a b}}\right) \frac{E_{n}(\{k, \epsilon, \sigma\})}{\sigma_{12} \ldots \sigma_{n 1}}, \\
& M_{n}=\int \frac{d^{n} \sigma}{\operatorname{volSL}(2, \mathbb{C})} \prod_{a}{ }^{\prime} \delta\left(\sum_{b \neq a} \frac{s_{a b}}{\sigma_{a b}}\right) E_{n}^{2}(\{k, \epsilon, \sigma\}),
\end{aligned}
$$

where $E_{n}(\{k, \epsilon, \sigma\})$ is a permutation-invariant function of momenta $k_{a}^{\mu}$, polarization vectors $\epsilon_{a}^{\mu}$, and $\sigma_{a}$. Note that the $\operatorname{SL}(2, \mathbb{C})$ invariance of the integrand constrains $E_{n}$ : under an $\operatorname{SL}(2, \mathbb{C})$ transformation, $\sigma_{a} \rightarrow\left(A \sigma_{a}+B\right) /\left(C \sigma_{a}+D\right)$, $E$ must transform as

$$
E_{n}(\{k, \epsilon, \sigma\}) \rightarrow E_{n}(\{k, \epsilon, \sigma\}) \prod_{a=1}^{n}\left(C \sigma_{a}+D\right)^{2} .
$$

It is also natural to expect that $E_{n}$ should be gauge invariant for each solution of the scattering equations.

At this point, it is worth to spell out how the measure is computed in practice, which uncovers a beautiful relation to a matrix found previously in the literature and, hence, shows its permutation invariance. Consider the object

$$
\int \frac{d^{n} \sigma}{\operatorname{volSL}(2, \mathbb{C})} \prod_{a} \delta \delta\left(\sum_{b \neq a} \frac{s_{a b}}{\sigma_{a b}}\right) \mathcal{I}
$$


where $\mathcal{I}$ represents either the integrand of Yang-Mills or that of gravity. Using a Fadeev-Popov procedure to gauge fix the $\operatorname{SL}(2, \mathbb{C})$ redundancy and, hence, fix the value of, say, $\sigma_{p}, \sigma_{q}, \sigma_{r}$, one finds that Eq. (6) becomes

$$
\int \prod_{c \neq p, q, r} d \sigma_{c}\left(\sigma_{p q} \sigma_{q r} \sigma_{r p}\right)\left(\sigma_{i j} \sigma_{j k} \sigma_{k i}\right) \prod_{a \neq i, j, k} \delta\left(\sum_{b \neq a} \frac{s_{a b}}{\sigma_{a b}}\right) \mathcal{I}
$$

The delta functions completely localize all integrals. As proven in Ref. [1], the scattering equations have $(n-3)$ ! solutions, and the answer is obtained by evaluating a Jacobian and the integrand on them. The Jacobian can be computed by starting with an $n \times n$ matrix $\Phi$ defined by

$$
\Phi_{a b}= \begin{cases}\frac{s_{a b}}{\left(\sigma_{a}-\sigma_{b}\right)^{2}}, & a \neq b \\ -\sum_{c \neq a} \frac{s_{a c}}{\left(\sigma_{a}-\sigma_{c}\right)^{2}}, & a=b .\end{cases}
$$

The fact that the delta functions exclude $\{i, j, k\}$ means that we have to delete those rows from $\Phi$ while having fixed the values of $\sigma_{p}, \sigma_{q}, \sigma_{r}$ means that we have to delete columns $\{p, q, r\}$. Let us denote the corresponding minor by $|\Phi|_{p q r}^{i j k}$. This minor is the Jacobian we are after. The answer is then

$$
\sum_{\{\sigma\} \in \text { solutions }} \frac{\left(\sigma_{p q} \sigma_{q r} \sigma_{r p}\right)\left(\sigma_{i j} \sigma_{j k} \sigma_{k i}\right)}{|\Phi|_{p q r}^{i j k}} \mathcal{I} \text {. }
$$

Precisely the combination that appears in this equation is what was called $\operatorname{det}^{\prime} \Phi$ by Cachazo and Geyer in Ref. [12] (inspired by a remarkable formula for maximally-helicityviolating gravity amplitudes found by Hodges in Ref. [13]) and which is known to be completely permutation invariant, i.e., independent of the choices made in selecting $\{i, j, k\}$ and $\{p, q, r\}$. More explicitly,

$$
\operatorname{det}^{\prime} \Phi:=\frac{|\Phi|_{p q r}^{i j k}}{\left(\sigma_{p q} \sigma_{q r} \sigma_{r p}\right)\left(\sigma_{i j} \sigma_{j k} \sigma_{k i}\right)} .
$$

Explicit form of $E_{n}(\{k, \epsilon, \sigma\})$. - In order to present the explicit form of $E_{n}(\{k, \epsilon, \sigma\})$, we first define the following $2 n \times 2 n$ antisymmetric matrix:

$$
\Psi=\left(\begin{array}{cc}
A & -C^{T} \\
C & B
\end{array}\right)
$$

where $A, B$, and $C$ are $n \times n$ matrices. The first two matrices have components

$A_{a b}=\left\{\begin{array}{ll}\frac{s_{a b}}{\sigma_{a}-\sigma_{b}}, & a \neq b \\ 0, & a=b,\end{array} \quad B_{a b}= \begin{cases}\frac{2 \epsilon_{a} \cdot \epsilon_{b}}{\sigma_{a}-\sigma_{b}}, & a \neq b \\ 0, & a=b,\end{cases}\right.$

while the third is given by

$$
C_{a b}= \begin{cases}\frac{2 \epsilon_{a} \cdot k_{b}}{\sigma_{a}-\sigma_{b}}, & a \neq b \\ -\sum_{c \neq a} \frac{2 \epsilon_{a} \cdot k_{c}}{\sigma_{a}-\sigma_{c}}, & a=b .\end{cases}
$$

The first important observation is that while the Pfaffian of $\Psi$ is zero, removing rows $i, j$, and columns $i, j$ with $1 \leq i<j \leq n$ gives rise to a new matrix $\Psi_{i j}^{i j}$ with nonzero Pfaffian and such that

$$
\operatorname{Pf}^{\prime} \Psi:=\frac{(-1)^{i+j}}{\left(\sigma_{i}-\sigma_{j}\right)} \operatorname{Pf}\left(\Psi_{i j}^{i j}\right)
$$

is independent of the choice of $i$ and $j$. We call $\mathrm{Pf}^{\prime} \Psi$ the reduced Pfaffian of $\Psi$.

The Pfaffian of $\Psi$ vanishes because its first $n$ rows (or columns) are linearly dependent: actually, the $n \times 2 n$ matrix $\left(A,-C^{T}\right)$ has two null vectors, $(1, \ldots, 1)$ and $\left(\sigma_{1}, \ldots, \sigma_{n}\right)$, thus, $(\operatorname{Pf} \Psi)^{2}=\operatorname{det} \Psi=0$. Now we turn to the proof that the reduced Pfaffian is invariant under permutations of particle labels. First note that simultaneous interchanges of two columns and rows change the sign of the Pfaffian. When exchanging two particle labels $a, b$, which are different from $i, j$, we must exchange rows and columns $a, b$ and also exchange $a+n, b+n$; when exchanging particle labels $i, j$, we only exchange $i+n$, $j+n$ in $\Psi_{i j}^{i j}$, and the additional minus sign cancels with the minus sign from the prefactor in the definition of $\mathrm{Pf}^{\prime} \Psi$. Hence, in both cases the reduced Pfaffian is invariant. Therefore, to prove permutation invariance, it suffices to prove that the reduced Pfaffian obtained from removing columns and rows 1,2 and that from removing 1, 3 are identical.

We multiply the first row and column of $\Psi_{12}^{12}$ by $\sigma_{13}$, and the first row and column of $\Psi_{13}^{13}$ by $\sigma_{12}$, and obtain two matrices we call $\Psi_{12}^{\prime 12}$ and $\Psi_{13}^{\prime 13}$. Next, we take a multiple of the $(i-2)$ th row and column of $\Psi_{12}^{\prime \prime 2}$ by $\sigma_{1 i}$ and add all the multiples to the first row and column, respectively, for $i=4, \ldots, n$; in this way, we obtain a new matrix $\Psi_{12}^{\prime \prime}$, and similarly, we have $\Psi_{13}^{\prime 13}$, whose Pfaffians are related to the original ones by $\operatorname{Pf} \Psi_{12}^{\prime \prime 12}=\sigma_{13} \operatorname{Pf} \Psi_{12}^{12}, \operatorname{Pf} \Psi_{13}^{\prime \prime 13}=\sigma_{12} \operatorname{Pf} \Psi_{13}^{13}$. By the scattering equations, it is straightforward to show that the first row and column of $\Psi_{12}^{\prime \prime 2}$ only differ from the first row and column of $\Psi_{13}^{\prime \prime} 13$ by a minus sign; note that other columns and rows of the two new matrices are identical, thus, $\operatorname{Pf} \Psi_{12}^{12} / \sigma_{12}=-\left(\operatorname{Pf} \Psi_{13}^{13}\right) /\left(\sigma_{13}\right)$. We conclude that the reduced $\operatorname{Pfaffian} \operatorname{Pf}^{\prime} \Psi$ is permutation invariant with respect to the particle labels.

Now we are ready to write down the proposal

$$
E_{n}(\{k, \epsilon, \sigma\})=\operatorname{Pf}^{\prime} \Psi(\{k, \epsilon, \sigma\}) .
$$

Combining this proposal for $E_{n}$ with the general formula (3) and (4) gives the main results of this Letter: a formula for the tree-level $S$-matrix of Yang-Mills in any dimension 


$$
A_{n}=\frac{1}{\operatorname{vol~SL}(2, \mathbb{C})} \int \frac{d^{n} \sigma}{\sigma_{12} \cdots \sigma_{n 1}} \prod_{a}{ }_{a} \delta\left(\sum_{b \neq a} \frac{s_{a b}}{\sigma_{a b}}\right) \operatorname{Pf}^{\prime} \Psi .
$$

And, using the KLT construction in the form discussed in Refs. [1,12] and the KLT orthogonality proven in Ref. [1], a formula for the tree-level $S$-matrix of gravity

$M_{n}=\frac{1}{\operatorname{volSL}(2, \mathbb{C})} \int d^{n} \sigma \prod_{a}{ }_{a} \delta\left(\sum_{b \neq a} \frac{s_{a b}}{\sigma_{a b}}\right) \operatorname{Pf}^{\prime} \Psi \operatorname{Pf}^{\prime} \tilde{\Psi}$

Here, $\tilde{\Psi}$ is taken to mean $\Psi(k, \tilde{\epsilon}, \sigma)$ and where $\tilde{\epsilon}_{a}$ represents the same physical polarization as $\epsilon_{a}$. In its simplest form, one can choose $\tilde{\epsilon}_{a}=\epsilon_{a}$ and obtain

$$
M_{n}=\frac{1}{\operatorname{vol~SL}(2, \mathbb{C})} \int d^{n} \sigma \prod_{a}^{\prime} \delta\left(\sum_{b \neq a} \frac{s_{a b}}{\sigma_{a b}}\right) \operatorname{det}^{\prime} \Psi,
$$

where $\operatorname{det}^{\prime} \Psi$ is $\operatorname{defined}$ as $\operatorname{det} \Psi_{i j}^{i j} / \sigma_{i j}^{2}$.

Finally, it is worth to also write both formulas in a form where all the integrals have been performed using Eqs. (9) and (10)

$$
A_{n}=\sum_{\{\sigma\} \in \text { solutions }} \frac{1}{\sigma_{12} \cdots \sigma_{n 1}} \frac{\operatorname{Pf}^{\prime} \Psi(\{k, \epsilon, \sigma\})}{\operatorname{det}^{\prime} \Phi}
$$

and

$$
M_{n}=\sum_{\{\sigma\} \in \text { solutions }} \frac{\operatorname{det}^{\prime} \Psi(\{k, \epsilon, \sigma\})}{\operatorname{det}^{\prime} \Phi} .
$$

Properties and checks.-Simple properties such as multilinearity in polarization vectors, $\operatorname{SL}(2, \mathbb{C})$ invariance, and its mass dimension are easy to check by using the expansion of the Pfaffian or its recursion relation (analogous to those of the determinant). Gauge invariance, as the statement that the amplitude vanishes when any $\epsilon_{a}^{\mu}$ is replaced by a multiple of $k_{a}^{\mu}$, is obvious since two columns of the matrix $\Psi$ and, hence, of $\Psi_{i j}^{i j}$ become multiples of each other under the replacement. More explicitly, assume that $\epsilon_{i}^{\mu}$ is replaced by $k_{i}^{\mu}$, then it can be easily seen that columns $i$ and $i+n$ of $\Psi$ become identical after realizing that

$$
C_{i i}=-\sum_{c \neq i} \frac{2 \epsilon_{i} \cdot k_{c}}{\sigma_{i}-\sigma_{c}} \rightarrow-\sum_{c \neq i} \frac{2 k_{i} \cdot k_{c}}{\sigma_{i}-\sigma_{c}}=0
$$

by the scattering equations. The last property which is manifest is the behavior under soft limits. As discussed in detail in Ref. [1], when we take $k_{n} \rightarrow 0, n-1$ of the scattering equations become identical to those of a system with $n-1$ particles. The last equation

$$
\sum_{b \neq n} \frac{s_{n b}}{\sigma_{n}-\sigma_{b}}=0
$$

becomes a polynomial for $\sigma_{n}$ of degree $n-3$ (due to momentum conservation). In this discussion, we focus on Yang-Mills amplitudes. It is convenient to compute $\mathrm{Pf}^{\prime} \Psi$ using $\Psi_{i j}^{i j}$ with $i \neq n, j \neq n$. The Pfaffian of a $2 m \times 2 m$ matrix $E$ satisfies a recursion relation of the form $\operatorname{Pf}(E)=\sum_{q=1}^{2 m}(-1)^{q} e_{p q} \operatorname{Pf}\left(E_{p q}^{p q}\right)$. Using this formula to expand $\operatorname{Pf} \Psi_{i j}^{i j}$ setting $p=n$, one finds that in the soft limit, only one term contributes and gives

$$
\operatorname{Pf} \Psi_{i j}^{i j} \rightarrow C_{n n} \operatorname{Pf} \Psi_{i j n(2 n)}^{i j n(2 n)} .
$$

Very nicely, $\operatorname{Pf} \Psi_{i j n(2 n)}^{i j n(2 n)}$ is independent of $k_{n}$ and $\epsilon_{n}$ and leads to $\operatorname{Pf}^{\prime} \Psi_{n-1}$, i.e., the reduced Pfaffian for $n-1$ particles. Using the explicit formula (16) in the soft limit, one finds

$$
A_{n} \rightarrow \sum_{i=1}^{(n-4) !} \oint_{\Gamma} d \sigma_{n} \frac{\sum_{a \neq n} \frac{2 \epsilon_{n} \cdot k_{a}}{\sigma_{n a}}}{\sum_{a \neq n} \frac{2 k_{n} \cdot k_{a}}{\sigma_{n a}}} \frac{\sigma_{n-1,1}}{\sigma_{n-1, n} \sigma_{n, 1}} \mathcal{I}_{n-1}^{(i)},
$$

where $\mathcal{I}_{n-1}^{(i)}$ are the terms in the expansion of Eq. (19) for $A_{n-1}$, and all $\sigma_{a}$ 's with $a \in\{1,2, \ldots, n-1\}$ are taken to be evaluated on the $i$ th solution. Also, the contour $\Gamma$ is defined to encircle the $n-3$ zeroes of the first factor in the denominator [14]. Using the residue theorem, one finds that there is no contribution at infinity, and only two poles have nonvanishing residue. These are at $\sigma_{n}=\sigma_{n-1}$ and at $\sigma_{n}=\sigma_{1}$. The residues are trivial to compute as only one term from the sum in the numerator and one from that in the denominator contribute, giving rise to

$$
A_{n} \rightarrow\left(\frac{\epsilon_{n} \cdot k_{n-1}}{k_{n} \cdot k_{n-1}}+\frac{\epsilon_{n} \cdot k_{1}}{k_{n} \cdot k_{1}}\right) A_{n-1},
$$

which is the correct soft behavior [15]. A completely analogous computation gives the correct soft behavior for gravity as well. Factorization of the amplitude on physical poles is a more involved computation and details are provided in Ref. [16].

We have also performed some nontrivial checks, such as the agreement of our formula for gluons with formulas available in the literature for three-, four-, and five-particle scattering in any dimension (see, e.g., Ref. [17] for $n=3,4$ and Ref. [18] for $n=5$ ). The case with five particles is the most interesting one as the scattering equations in dimensions greater than four do not factor, and the two solutions come from an irreducible quadratic equation. This is the first case that our formula clearly computes the amplitudes in a novel way. We also performed numerical checks that when evaluated in four-dimensional kinematics, our formula reproduces all amplitudes with $n \leq 8$ and in all possible helicity sectors (including the all plus and all but one plus). 
Discussion.-We have presented a formula for the complete tree-level $S$-matrix of gluons and gravitons in any spacetime dimension. While formulas in dimensions less than ten could exploit the presence of supersymmetry in defining an on-shell superspace, such as the formula of Witten and of Roiban, Spradlin, and Volovich does in four dimensions [19], our formula necessarily depends on polarization vectors as it is also valid in dimensions where supersymmetry does not exist. Any formula which contains polarization vectors must satisfy the constraint that it vanishes when any polarization vector is replaced by a multiple of its momentum vector. What we have found in this work is that there exists a very compact formula in which gauge invariance is actually a simple property of its intrinsic structure, and, indeed, it was the main clue for its derivation.

As discussed in Ref. [20] and more recently in Refs. [1,21], there are compact formulas for string amplitudes in terms of Yang-Mills or gravity amplitudes, and our proposal here also provides a simple representation of string amplitudes in terms of polarization vectors. In relation to string amplitudes, it is important to mention an intriguing connection to their high energy scattering limit. In the work of Gross and Mende [4], the scattering equations appear as the conditions imposed by the saddle point evaluation of the string amplitude. It is tempting to suggest that this is more than a coincidence.

Finally, also worth mentioning is that all $(n-3)$ ! solutions of the scattering equations give rise to gaugeinvariant contributions. Moreover, under factorization limits, each term in Eqs. (19) and (20) either develops a pole and "factors," or it remains finite. This is reminiscent of the behavior of partial amplitudes in Yang-Mills theory where a decomposition of the full amplitude is made in parts that do not exhibit all factorization channels. Adding the fact that in dimensions greater than four the $(n-3)$ ! do not split into sectors, it is natural to suggest that each solution is a "partial amplitude." In Yang-Mills theory, this decomposition is in addition to the usual color ordering one, while in gravity, it is all there is. It would be fascinating to fully uncover the physical meaning of this new decomposition.

This work is supported by Perimeter Institute for Theoretical Physics. Research at Perimeter Institute is supported by the Government of Canada through Industry Canada and by the Province of Ontario through the Ministry of Research \& Innovation.

Note added.-Recently, more detailed discussions of our prescription and its application in scalar theories were summarized in Ref. [22], the validity of our formula was proven by Ref. [23] using Britto-Cachazo-Feng-Witten recursion relations [24,25], and ambitwistor string models were constructed in Ref. [26] that produce our formula. *fcachazo@perimeterinstitute.ca

† she@perimeterinstitute.ca

†yyuan@perimeterinstitute.ca

[1] F. Cachazo, S. He, and E. Y. Yuan, arXiv:1306.6575.

[2] The KLT orthogonality proved in Ref. [1] is the fact that "Parke-Taylor" vectors evaluated on distinct solutions of the scattering equations are orthogonal with respect to the kernel of the field-theory KLT relations, which relate Yang-Mills and gravity amplitudes [27].

[3] D. Fairlie and D. Roberts (unpublished); D. Roberts, Ph.D. thesis, Durham University, 1972; D. B. Fairlie, Adv. Theor. Math. Phys. 2009, 284689 (2009).

[4] D. J. Gross and P. F. Mende, Nucl. Phys. B303, 407 (1988).

[5] E. Witten, Adv. Theor. Math. Phys. 8, 779 (2004).

[6] Y. Makeenko and P. Olesen, Phys. Lett. B 709, 285 (2012).

[7] F. Cachazo, arXiv:1206.5970.

[8] Z. Bern, J. J. M. Carrasco, and H. Johansson, Phys. Rev. D 78, 085011 (2008).

[9] The equations in Ref. [7] and those in Eq. (1) are related by the $\operatorname{SL}(2, \mathbb{C})$ transformation $S=\left(\begin{array}{cc}0 & 1 \\ -1 & 0\end{array}\right)$.

[10] N. E. J. Bjerrum-Bohr, P. H. Damgaard, and P. Vanhove, Phys. Rev. Lett. 103, 161602 (2009).

[11] S. Stieberger, arXiv:0907.2211.

[12] F. Cachazo and Y. Geyer, arXiv:1206.6511.

[13] A. Hodges, arXiv:1204.1930.

[14] Since $\sigma_{a}$ 's are taken to be complex numbers in this Letter, the delta functions imposing the scattering equations are, in fact, poles, and all our integrals are contour integrals.

[15] S. Weinberg, Phys. Rev. 135, B1049 (1964); Phys. Rev. 140, B516 (1965).

[16] F. Cachazo, S. He, and E. Y. Yuan, follow the link: Supplementary notes, http://ellisyeyuan.wordpress.com/ 2013/07/07/soft-limits-and-factorizations/ (2013).

[17] M. B. Green, J. Schwarz, and E. Witten, Superstring Theory, Cambridge Monographs on Mathematical Physics Vol. 1 (Cambridge University Press, Cambridge, England, 1987).

[18] R. Medina, F. T. Brandt, and F. R. Machado, J. High Energy Phys. 07 (2002) 071.

[19] E. Witten, Commun. Math. Phys. 252, 189 (2004); R. Roiban, M. Spradlin, and A. Volovich, Phys. Rev. D 70, 026009 (2004).

[20] C. R. Mafra, O. Schlotterer, and S. Stieberger, Nucl. Phys. B873, 419 (2013); B873, 461 (2013).

[21] S. Stieberger and T. R. Taylor, Nucl. Phys. B873, 65 (2013); Phys. Lett. B 725, 180 (2013).

[22] F. Cachazo, S. He, and E. Y. Yuan, J. High Energy Phys. 07 (2014) 033.

[23] L. Dolan and P. Goddard, J. High Energy Phys. 05 (2014) 010 .

[24] R. Britto, F. Cachazo, and B. Feng, Nucl. Phys. B715, 499 (2005); R. Britto, F. Cachazo, B. Feng, and E. Witten, Phys. Rev. Lett. 94, 181602 (2005).

[25] N. Arkani-Hamed and J. Kaplan, J. High Energy Phys. 04 (2008) 076.

[26] L. Mason and D. Skinner, J. High Energy Phys. 07 (2014) 048; N. Berkovits, J. High Energy Phys. 03 (2014) 017.

[27] H. Kawai, D. Lewellen, and S. Tye, Nucl. Phys. B269, 1 (1986). 